\title{
Study on the substation inspection robot navigation system based on Fuzzy-PID control Suiqun $\mathrm{LI}^{1, \mathrm{a}}$; Lecai $\mathrm{CAl}^{2, \mathrm{~b}}$; Xiang Gao ${ }^{1, \mathrm{c}}$; Yong Wang ${ }^{1}$; Yonghong Zhu ${ }^{1}$ \\ ${ }^{1}$ School of SI Chuan University of Science \& Engineering, ZI Gong 643000, China; \\ ${ }^{2}$ School of YI BIN University, YI BIN 644000, China. \\ asuiqun17@qq.com, bCLC@suse.edu.cn, c1326820449@qq.com
}

Keywords: magnetic navigation, patrol robot, fuzzy PID control.

\begin{abstract}
Based appear for magnetic navigation system for substation inspection robot navigation course accumulation of errors phenomenon, fuzzy PID control algorithm is applied to inspection robot navigation system, navigation path through the logic of correction, to achieve stability navigation. On the movement patterns of inspection robot is modeled using fuzzy algorithm parameters that affect the stability of the navigation processing, the simulation shows that fuzzy -PID robot navigation algorithm higher precision control, better operational stability.
\end{abstract}

\section{Introduction}

High-voltage substation has the characteristics of complex work environment, high risk factor, and the stable development of the national economy and the life. Monitoring is especially important for high-voltage substations, manual monitoring in high voltage and strong electromagnetic environment has some limitations, and substation inspection robot can make up for lack of manual monitoring.

As an intelligent mobile robot, navigation is the basic guarantee for the completion of the inspection task. The navigation system is based on the magnetic navigation system design ${ }^{[1]}$. Magnetic navigation, is the robot's front mounted magnetic sensor array to detect the relative magnetic track of the robot relative to the magnetic track, the speed difference between the two driving wheels to control its along the magnetic track ${ }^{[2]}$.

\section{The vehicle body structure design and model establishment of magnetic navigation system}

\subsection{Design of the car body structure of the gear $\operatorname{train}^{[3]}$}

The two drive wheels arranged on the chassis, the vehicle body is fixed to the chassis, this layout makes navigating correction inspection robot is completely under the control of the drive motor is completed, the drive motor and the drive wheel is connected to nature the difference is the speed of the drive motor speed operation control two drive wheels, thus ensuring inspection robot center is always in the top of the route ${ }^{[4]}$. Figure 1 is the gear train layout structure of inspection robot.

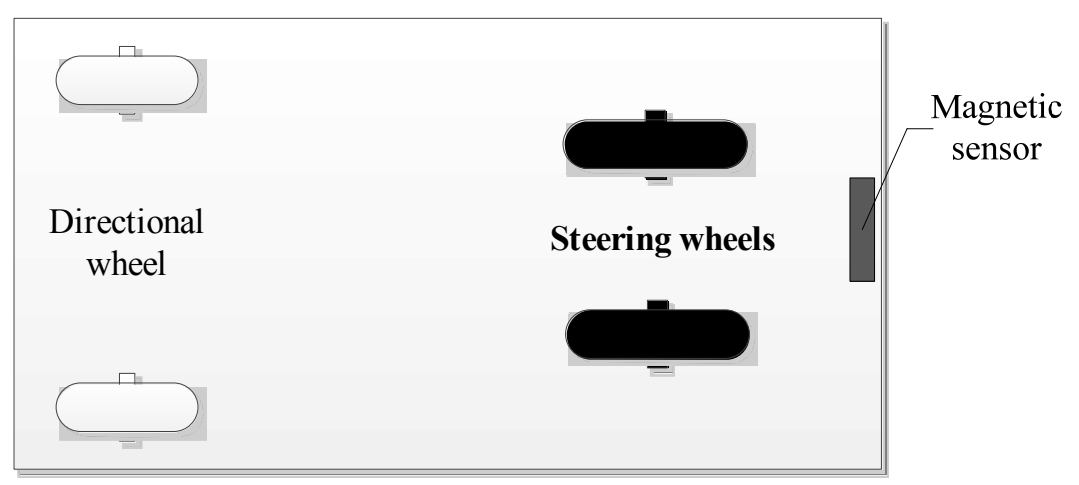

Fig. 1 Gear train layout structure of inspection robot 
2.2 Establishment of magnetic navigation system model

In the direction of $\mathrm{Y}$-axis magnetic stripe pavement, perpendicular to the direction of the magnetic stripe of the $\mathrm{X}$-axis, to the intersection of the axis of the rear magnetic stripe patrol robot coordinate origin, establish inspection robot coordinate system. Figure 2 is a schematic view of the inspection robot heading model, dots represent the magnetic sensor sampling points, uniform distribution of magnetic sensors spaced $1 \mathrm{~cm}$. By inspection robot detects the front end of the magnetic sensor offset $\mathrm{D}$, inspection robot body length $\mathrm{L}$, it is possible to calculate the angle a of inspection robot and central offset $b$. The mathematical relationship is as follows:

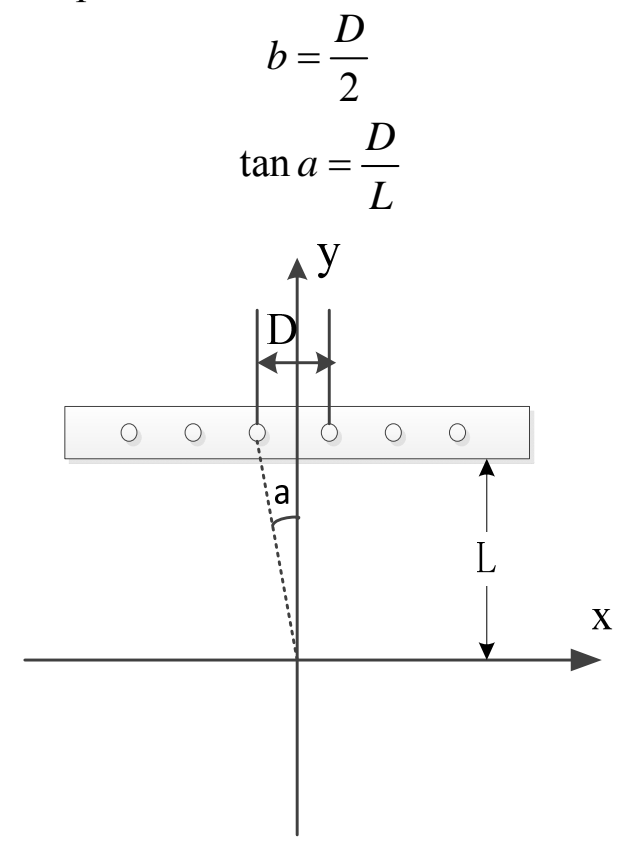

Fig. 2 Schematic model of inspection robot heading

The magnetic sensor according to the sampling data, the central controller can be calculated with angle and horizontal angle, and then draw the corresponding control information through the design of algorithms, the control information is transmitted to the motor drivers, motor drivers by controlling the right and left motors' speed to achieve correct direction. By repeating this process running, inspection robot can automatically navigate ${ }^{[5]}$.

\section{Fuzzy -PID control algorithm}

To achieve PID control algorithm must accurately understand the Mathematical model and PID control algorithm to change the system of control of nonlinear effect is not ideal, and therefore a fuzzy control algorithm. Fuzzy control algorithm mainly through the case of field staff or experts do not need to know the exact number of the controlled object, with a wealth of operational experience to control a number of complex operations, and then to review and summarize the experience and knowledge of building a knowledge model If the idea of fuzzy mathematics with which to quantify the formation of fuzzy control algorithm ${ }^{[6]}$. Compared with conventional PID control, fuzzy control system is characterized by:

(1) in the design of fuzzy control system, it cannot need to know the exact mathematical model of the controlled object, but it must have a full understanding of the characteristics of the controlled object.

(2) the mathematical variable can be substituted by linguistic variables, and the expert knowledge base can be constructed by the combination of the two.

(3) compared with the conventional control, the robustness is strong, and it has obvious advantages for solving nonlinear, multi disturbance, time-varying and multi - level delay systems.

The key factor of fuzzy controller is fuzzy control rules, the fuzzy control rules are mainly based on the practical experience of experts or operators. The error and the change rate as the input 
variables of the fuzzy controller, the output variable of the fuzzy controller, the control variables U.

Fuzzy -PID control is based on the classic PID control, the control principle is the rate of change of the error and error correction. Therefore, fuzzy -PID control by fuzzy control module and the classic PID control module, as shown in Figure 3.

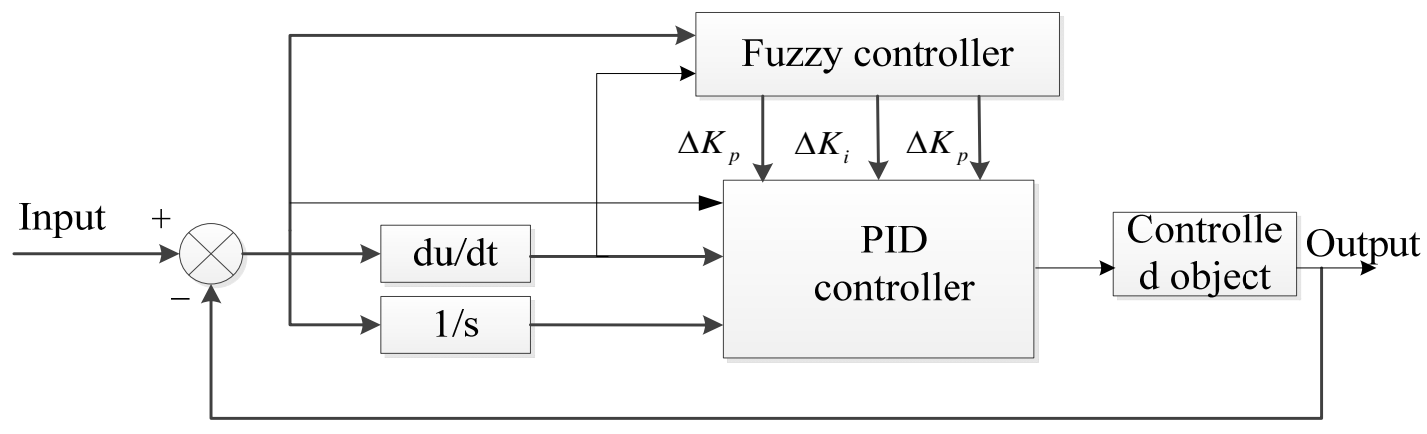

Fig. 3 Fuzzy -PID control structure diagram

\section{Determination of fuzzy-PID control rules ${ }^{[7]}$}

When the inspection robot is running, at first the robot may be able to correctly detect a predetermined path, but due to an error between the motor, transmission errors and wheel slippage and other factors will lead to the inspection robot may deviates from the predetermined trajectory in running time . As mentioned above, the output signal of the magnetic sensor monitoring point inspection robot compartment $1 \mathrm{~cm}$, magnetic stripe width is $3 \mathrm{~cm}$. The initial state is magnetic sensor in the middle of the two detection point is 9 and 10 detection point and the center line of the navigation magnetic stripe center line coincide. According to the layout of the magnetic sensor detection, can detect the body axis and the magnetic deviation angle and body position deviation.

The position deviation of the inspection robot the mathematical relationship between $e$ and magnetic sensor for magnetic deviation:

$$
e=\frac{D}{2}
$$

The mathematical relationship between the angle $a$ and position deviation:

$$
a=\arctan \frac{2 e}{L}
$$

And, $\mathrm{L}$ is the length of the body.

Therefore, the basic theory of position deviation $e$ is $[-45 \mathrm{~mm},+45 \mathrm{~mm}]$, According to inspection robot magnetic sensor detects the range of signal, the magnetic sensor and the path of the angle deviation $a$ set in $\left[-10^{\circ},+10^{\circ}\right]$, The fuzzy domain of distance deviation $e$ and the angle deviation $a$ are taken as $\{-3,-2,-1,0,1,2,3\}$.

Fuzzy -PID control system is composed of two parts of adjustable parameters PID controller and fuzzy controller. The initial value of fuzzy -PID control system is the inspection robot's positional deviation $e$ and the robot body angle deviation $a$, the system shown in Figure 4 .

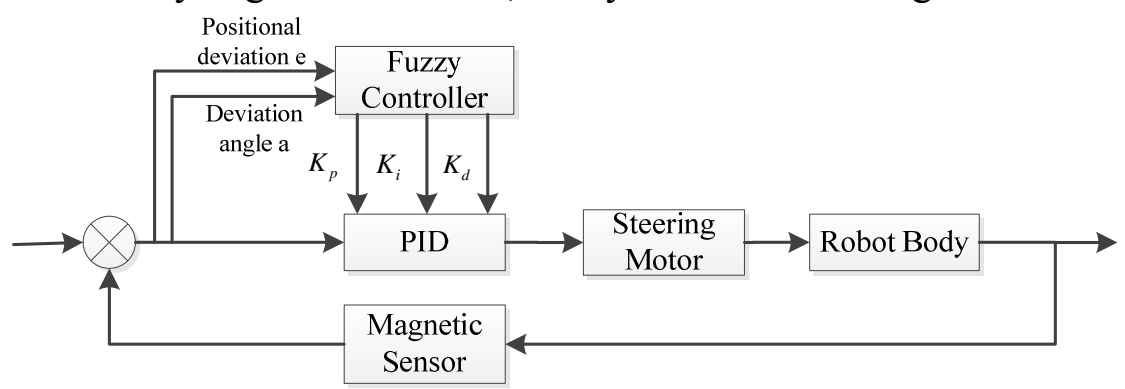

Fig. 4 Fuzzy -PID control system diagram 


\section{Fuzzy -PID control of Simulink simulation ${ }^{[8]}$}

In order to realize the simulation of the fuzzy -PID control system in Simulink, the control module of the adjustable PID parameters is needed. Design of the PID controller module with adjustable parameters by the position PID control algorithm. Position PID control algorithm is as follows.

$$
u(k)=K_{p} E(k)+K_{i} \sum_{i=0}^{k} E(i)+K_{d} E_{c}(k)
$$

In the Simulink environment ${ }^{[9]}$, the fuzzy -PID control simulation is established as figure 5.

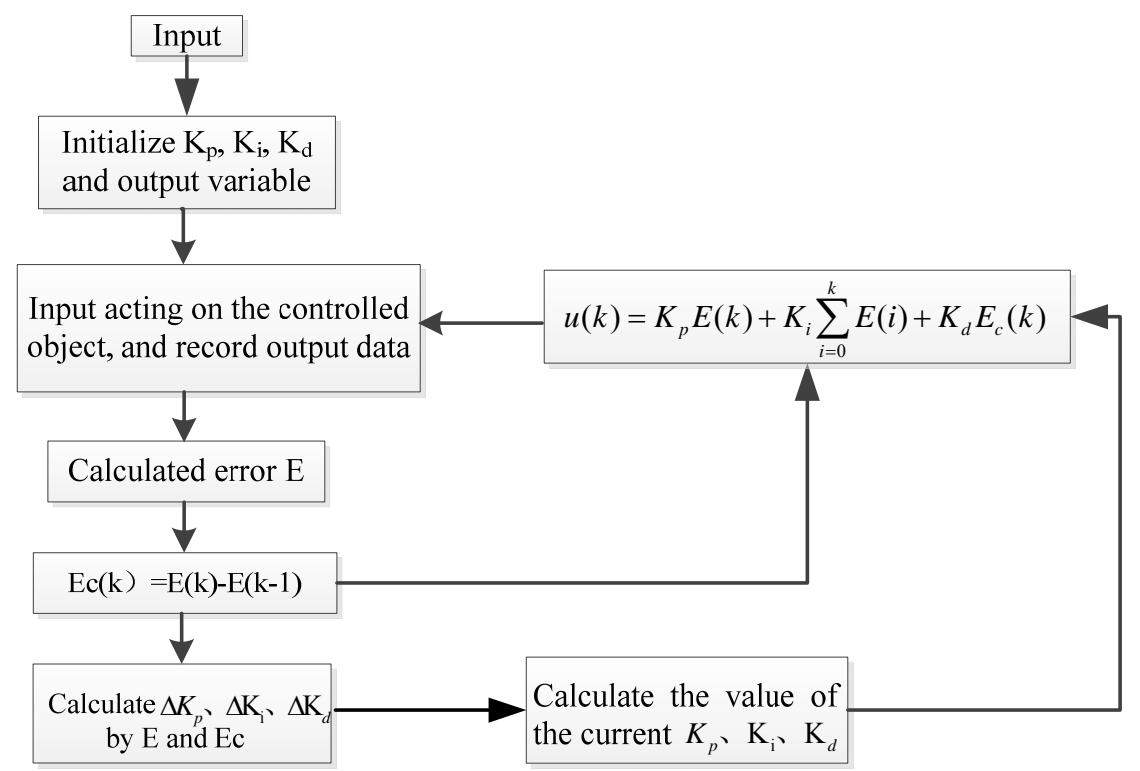

Fig. 5 simulation flow chart

The step response simulation of the classical PID control and fuzzy -PID control is as follows:
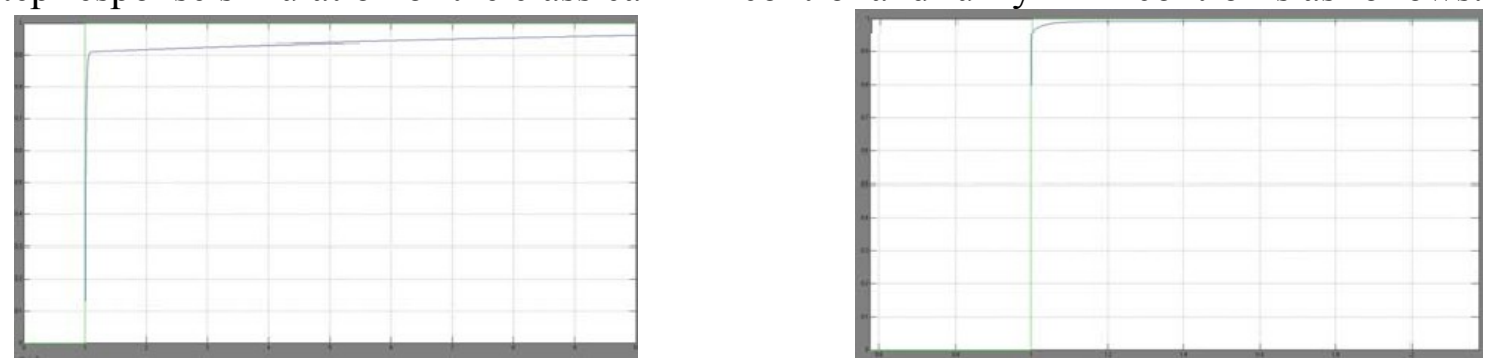

Fig.6 step response curve of classical PID control Fig. 7 step response curve of fuzzy -PID control

From the above two graphs, the fuzzy -PID control is relatively stable, while the steady error of the classical PID control system is larger. Adjustment time is about 1s, there is no super regulation, and the response speed of the fuzzy -PID control system is significantly faster, which is because the initial parameters of the classical PID control system cannot be changed at will, and the fuzzy -PID control system can realize the real-time on-line auto tuning of control parameters.

The paper also made a vague Fuzzy-PID immunity testing, the test method of control is added 0.1 of interference information at $\mathrm{t}=1.5 \mathrm{~s}$, comparison chart to get the following response:

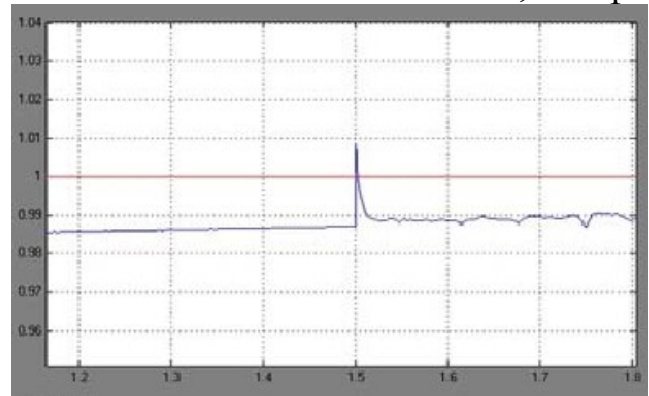

Fig.8 PID control and interference response curve

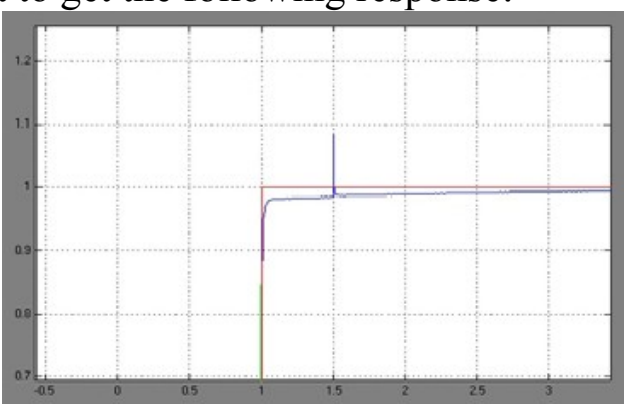

Fig. 9 Fuzzy -PID control response curve 
When the classic PID control to join 0.1 of the interference information, the error is about 0.1 , the same interference information to join the fuzzy -PID control system, the error is very small, about 0.01 . It can be concluded that the anti disturbance ability of fuzzy -PID control algorithm is better than the classical PID algorithm, and the robustness is better.

\section{Summary}

Through the simulation analysis, it is concluded that the fuzzy -PID controller can improve the stability of navigation control system, and the system has strong anti-interference ability, better robustness and better control effect.

\section{Acknowledgments}

Thank the teachers who give me a careful guidance in the process of writing and modifying my paper. Thank the students who give me an enthusiasm help in the process of data collection.

Fund: Graduate student innovation fund project in Sichuan University of Science and Engineering (y2014002); Internet of things technology and application of Sichuan Province Youth Science and technology innovation team(2011JTD0031); Research and development of automatic target tracking system based on machine vision(2013RZY03).

Corresponding author: Suiqun Li, master, major in research and application of network technology from the things, E-mail:suiqun17@qq.com.

\section{References}

[1] Wang Yuanzhe, Hu Hongjie. Design and implementation of magnetic navigation of intelligent vehicle system [J]. Automation Instrumentation . 2011,11:5-8.

[2] Li Qiang, Hu Ze, Ge Liang. The use of electromagnetic navigation AGV design [J]. Modern Electronic Technology, 2012,12:79-81.

[3] Yao Yi,Chen Guangjian, Jia Jinling. Research on robot path planning based on fuzzy neural network algorithm [J]. Journal of Sichuan University of Science and Engineering (NATURAL SCIENCE EDITION), 2014,06:30-33.

[4] Xu Huahua, Xu Ying, Zheng Wenfeng, Hu Xiaoping. Control system design of wheeled mobile robot [J]. Machinery and Electronics, 2009,02:67-69.

[5] Li Jian, Wang Dongqing, Wang Limei. Design of fuzzy PID controller and MATLAB simulation [J]. Industrial Control Computer, 2011,05:56-57+95.

[6] Zhang Jing Zhou, Wei Jing Yang, Zhang Anxiang. Fuzzy adaptive PID control research and simulation application [J]. Computer Simulation.2009,09:132-135+163.

[7] Gao Daoxiang, Xue Dingyu. System simulation study of robot robust adaptive control system based on MATLAB/Simulink [J]. Journal of System Simulation, 2006,07:2022-2025.

[8] Wang Suqing, Jiang Weifu. PID parameters' tuning based on MATLAB/Simulink[J]. Automation Technology and Application, 2009,03:24-25+28.

[9] Li Xiaohong, YaoRao, Liu Song qing. Design and simulation of fuzzy PID parameters' self tuning controller based on Simulink[J]. Engineering of Jilin Normal University Journal, 2006,09:33-37. 